\title{
BioBarcode: a general DNA barcoding database and server platform for Asian biodiversity resources Jeongheui $\mathrm{Lim}^{1}$, Sang-Yoon Kim ${ }^{1}$, Sungmin Kim${ }^{2}$, Hae-Seok Eo ${ }^{3}$, Chang-Bae Kim ${ }^{4}$, Woon Kee Paek ${ }^{5}$, Won Kim ${ }^{* 2}$ and Jong Bhak*1
}

Addresses: ${ }^{1}$ Korean BioInformation Center, Korea Research Institute of Bioscience and Biotechnology, Daejeon, 305-806, Korea, ${ }^{2}$ School of Biological Science, Seoul National University, Seoul, 151-742, Korea, ${ }^{3}$ School of Computational Sciences, Korea Institute for Advanced Study, Seoul, 130-722, Korea, ${ }^{4}$ Department of Life Science, Sangmyung University, Seoul, 110-743, Korea and ${ }^{5}$ Division of Natural History, National Science Museum, Daejeon, 305-705, Korea

E-mail: Jeongheui Lim - jhlim@kribb.re.kr; Sang-Yoon Kim - sykim@kribb.re.kr; Sungmin Kim - kandury@hanmail.net; Hae-Seok Eo - ehs0328@kias.re.kr; Chang-Bae Kim - evodevo@smu.ac.kr; Woon Kee Paek - paekwk@mest.go.kr; Won Kim* - wonkim@plaza.snu.ac.kr; Jong Bhak* - jongbhak@yahoo.com

${ }^{*}$ Corresponding author

from Asia Pacific Bioinformatics Network (APBioNet) Eighth International Conference on Bioinformatics (InCoB2009)

Singapore 7-II September 2009

Published: 3 December 2009

BMC Genomics 2009, I0(Suppl 3):S8 doi: 10.1186/1471-2164-10-S3-S8

This article is available from: http://www.biomedcentral.com/I47/-2/64//0/S3/S8

(c) 2009 Lim et al; licensee BioMed Central Ltd.

This is an open access article distributed under the terms of the Creative Commons Attribution License (http://creativecommons.org/licenses/by/2.0), which permits unrestricted use, distribution, and reproduction in any medium, provided the original work is properly cited.

\begin{abstract}
Background: DNA barcoding provides a rapid, accurate, and standardized method for specieslevel identification using short DNA sequences. Such a standardized identification method is useful for mapping all the species on Earth, particularly when DNA sequencing technology is cheaply available. There are many nations in Asia with many biodiversity resources that need to be mapped and registered in databases.

Results: We have built a general DNA barcode data processing system, BioBarcode, with open source software - which is a general purpose database and server. It uses mySQL RDBMS 5.0, BLAST2, and Apache httpd server. An exemplary database of BioBarcode has around 11,300 specimen entries (including GenBank data) and registers the biological species to map their genetic relationships. The BioBarcode database contains a chromatogram viewer which improves the performance in DNA sequence analyses.

Conclusion: Asia has a very high degree of biodiversity and the BioBarcode database server system aims to provide an efficient bioinformatics protocol that can be freely used by Asian researchers and research organizations interested in DNA barcoding. The BioBarcode promotes the rapid acquisition of biological species DNA sequence data that meet global standards by providing specialized services, and provides useful tools that will make barcoding cheaper and faster in the biodiversity community such as standardization, depository, management, and analysis of DNA barcode data. The system can be downloaded upon request, and an exemplary server has been constructed with which to build an Asian biodiversity system http://www.asianbarcode.org.
\end{abstract}




\section{Background}

DNA barcoding is the standardized minimal approach to facilitate biodiversity studies that include species identification and discovery. It helps researchers to understand evolutionary and genetic relationships by assembling molecular, morphological, and distributional data [1]. Species-level identification through DNA barcoding is usually accomplished by the retrieval of a short DNA sequence from a standard part of the genome (i.e., 650base fragment of the $5^{\prime}$ end of the mitochondrial cytochrome $c$ oxidase I (COI) gene for animal species) from the specimen under investigation [2]. The barcode sequence from each unknown specimen is then compared with a library of reference barcode sequences derived from individuals of known identity [3].

The Consortium for the Barcode of Life (CBOL), which was launched in May 2004 and now includes more than 170 member organizations from 50 countries, is promoting DNA barcoding sensu stricto as the global standard for biological identification [4]. In contrast with the limited supply of taxonomic expertise, the need to assign specimens to known species arises every day and everywhere. Using molecular biomarkers, nonspecialists can assign specimens to known species - even specimens that can confound specialists (e.g., eggs, larvae, incomplete adults). Barcoding can therefore free taxonomists from the routine identification task of documenting new species. Next-generation DNA sequencing systems [5,6] will enable the rapid production of barcodes, thus eventually promoting the assignment of unknown individuals to classified species.

DNA barcoding sensu lato have reached out actively to new research areas other than taxonomy such as forensic science $[7,8]$, the biotechnology and food industries, and animal diet $[9,10]$. Ecologists, environmental scientists, agricultural inspectors, public health officials, and other potential users with the need to identify specimens are exploring barcoding as a new approach to applied problems [11]. Taxon identification with diagnostic single-nucleotide polymorphisms (SNPs) and biodiversity assessment from environmental samples (e.g., soil and water) can also be considered DNA barcoding sensu lato [12].

The DNA barcoding pilot projects contain several large groups of animals such as birds [13], fish [14], cowries [15], spiders [16], amphibians [17], and several arrays of Lepidoptera [18-20]. In addition, DNA barcoding systems are now being established for other groups of organisms, including plants [21], macroalgae [22], fungi [23], protists [24], and bacteria [25]. The barcoding projects of Korea Barcode of Life (KBOL), launched in April of 2007, are currently collecting barcode data of vertebrates [26], invertebrates, land tracheophytes, and lower plants.

We built a DNA barcoding database and web server system, BioBarcode, which was developed as a part of $\mathrm{KBOL}$ project, to provide a reusable barcode construction system for more specific projects. In other words, BioBarcode is a bioinformatics template or platform rather than a specific DNA barcode server. The purpose of BioBarcode is to be used by biologists who have specific species information and want to build a DNA barcode database and server. It supports the compilation, storage, analysis, and publication of high-quality DNA barcode records. For many experimental biologists, building a local DNA barcoding system is expensive and time consuming. Therefore, BioBarcode will be useful for providing the tools needed to launch successful barcoding projects in the Asian biodiversity research community, including software for data management and analysis, data standards, and a data repository. To establish data standard, we have adopted the guidelines from CBOL and GenBank at the National Center for Biotechnology Information (NCBI) that must be satisfied for records to gain formal barcode status. Furthermore, it can be used for promoting international collaboration for building an Asian biodiversity system aiming to be the Asian biodiversity database server. Here, we introduce an exemplary web system using BioBarcode.

\section{Methods}

\section{System architecture and scheme}

The database structure of BioBarcode system consists of 12 tables as shown in Figure 1. These tables are mainly bc_entry (a table for management of general information on specimens), and following taxonomy, project, location, attached files (image and chromatogram), and a member management table (Table 1). We used a lineage table as tax_names and tax_nodes from the NCBI taxonomy database to search and navigate lineage. This table contains taxonomy information of 370,000 species and their lineages from kingdom to subspecies, therefore, users can choose taxonomy and lineage information by keyword suggestion. The tables of image and trace files, such as bc_file_img and bc_file_trace, were separated to speed up and manage attachments easily. Minimal information is required for member registration, and we accept various types of geographical information such as addresses, latitude and longitude positions on Google map, and zipcodes (this zipcode data is only available in South Korea). These are implemented using mySQL RDBMS 5.0 as DBMS.

\section{Data uploads and repository}

Anyone can create a project(s) by registering as a BioiBarcode user through the completion of a short 


\begin{tabular}{|l|l|}
\hline \multicolumn{2}{|c|}{ bc_member } \\
\hline PK & id \\
\hline & $\begin{array}{l}\text { user_id } \\
\text { user_name } \\
\text { password } \\
\text { email } \\
\text { created } \\
\text { last_login } \\
\text { org } \\
\text { phone } \\
\text { ur_1 } \\
\text { ur_2 } \\
\text { FK1 } \\
\text { comments } \\
\text { eid }\end{array}$ \\
\hline
\end{tabular}
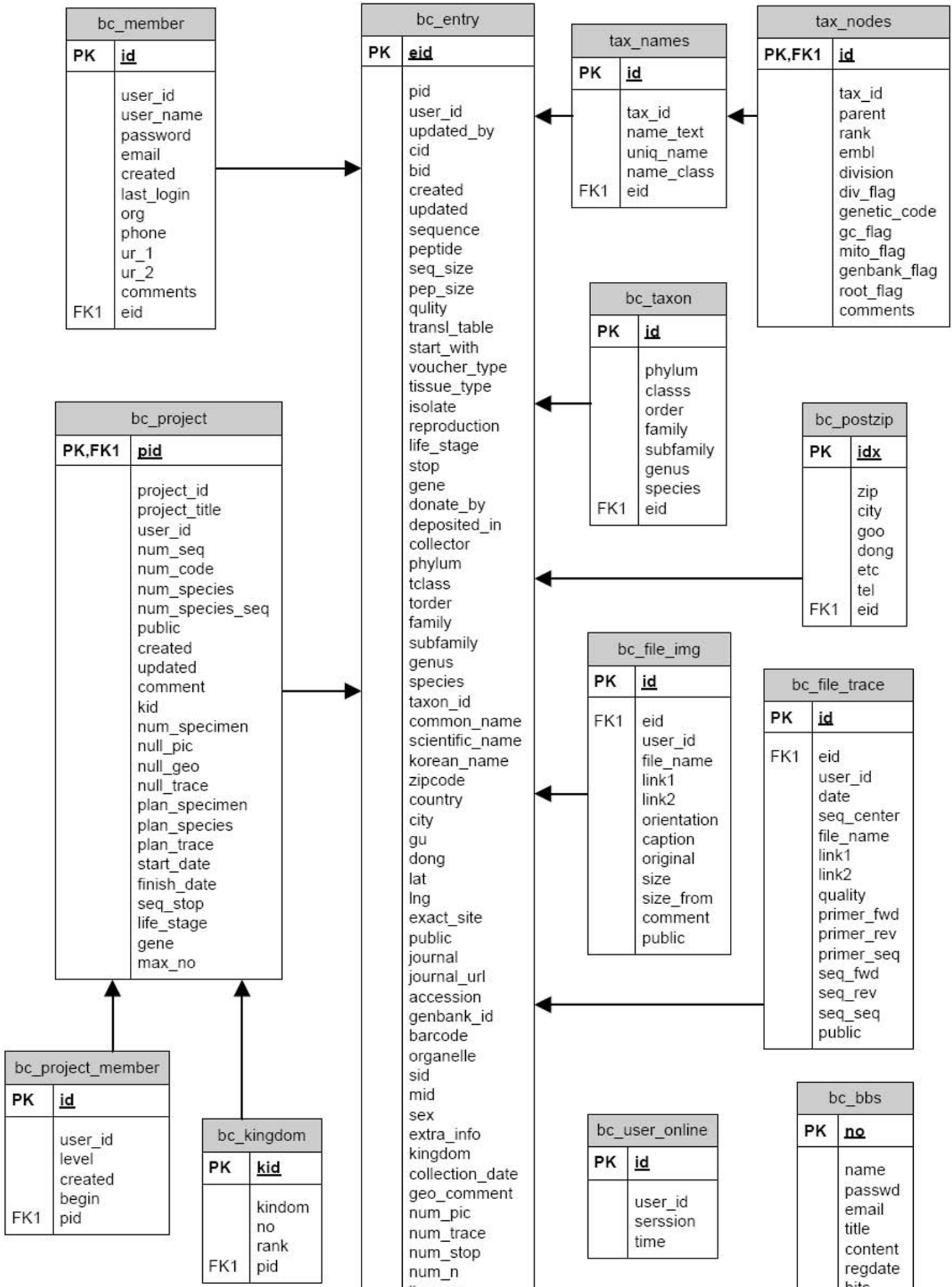

sequen
peptide
seq siz

pep_size

qulity

transl_table

start_with

voucher type

tissue_type

isolate

reproduction

life_stage

stop

gene

donate_by

deposited_in

collector

phylum

tclass

torder

family

subfamily

genus

species

taxon_id

common_name

scientific_name

korean_name

zipcode

country

city

gu

dong

lat

Ing

exact_site

public

journal

journal_url

accession

genbank_id

barcode

organelle

sid

mid

sex

extra info

kingdom

collection_date

geo comment

num_pic

num trace

num_stop

num n

lineage
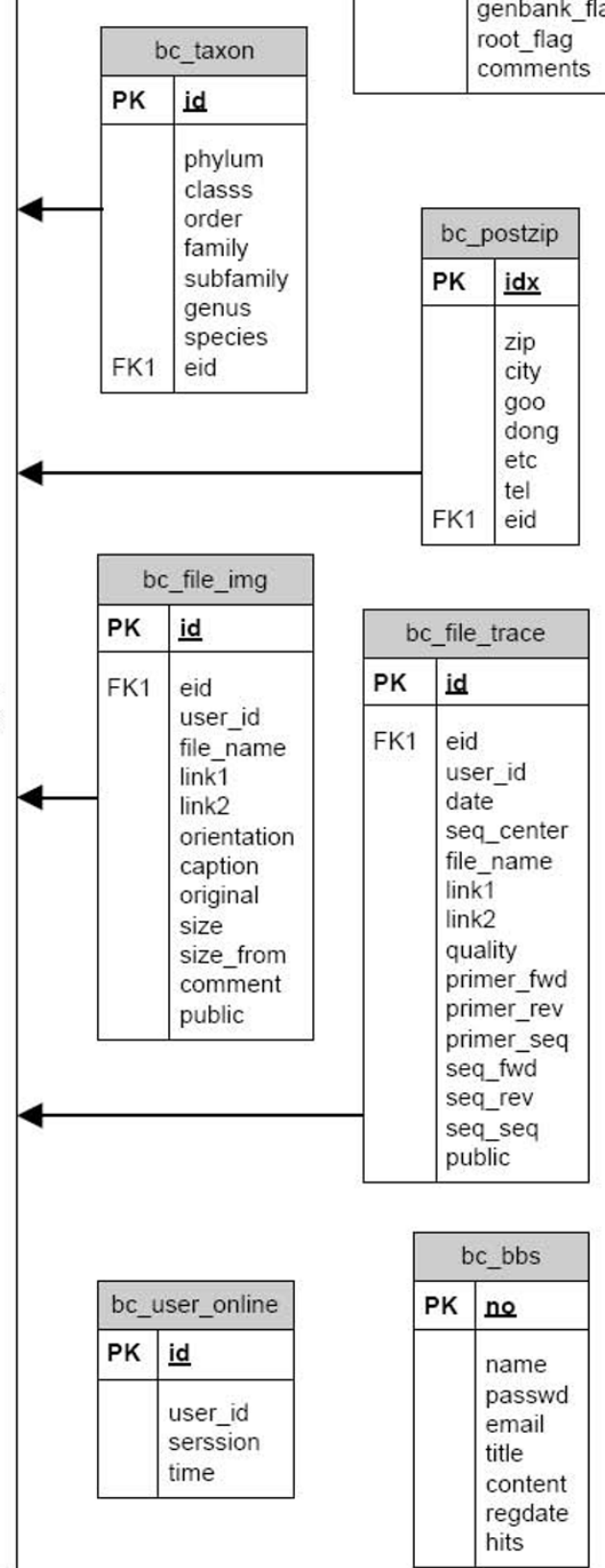

Figure I

BioBarcode system structure. The BioBarcode database consists of I 2 tables and implemented using mySQL RDBMS 5.0 as DBMS. The tables of image and trace files were separated to speed up and manage attachments easily. 
Table I: Tables used in the BioBarcode database

\begin{tabular}{ll}
\hline Table Name & \multicolumn{1}{c}{ Function } \\
\hline bc_taxon & Taxonomy information \\
tax_names & Taxonomy Name \\
tax_nodes & Taxonomy Node \\
bc_project_manager & Project Management \\
bc_kingdom & Project Classification \\
bc_project & Project Information \\
bc_postzip & Postal Address and ZIP code (geographical \\
& information) \\
bc_entry & Entry (general information of specimens) \\
bc_file_trace & Attached chromatogram file \\
bc_file_img & Attached Image File \\
bc_member & Registered Member Management \\
bc_bbs & Bulletin Board System for News \\
\hline
\end{tabular}

The BioBarcode database tables are mainly bc_entry (general information of specimens), and following taxonomy, project, location, attached files (images, chromatogram) and a member management table.

online form http://www.asianbarcode.org/register.php. While the data upload of the Barcode of Life Data Systems (BOLD, http://www.barcodinglife.org) is carried out in two parts of specimen and sequence, BioBarcode system can be uploaded data in three parts: specimen, taxonomy, and sequence.

Key specimen data, such as voucher information and collection data with geographical distributions, is recorded in the specimen entry. Clicking an icon using the Google map API makes an automatic entry of longitude and latitude of the selected location in geographical information (Figure 2). In the taxonomy entry, partial information on a species, such as full taxonomy, lineage, taxon ID (linked with NCBI Taxonomy database), common name, scientific name, and Korean name, is input. In the sequence entry, the primary barcode information is DNA which is automatically translated to protein when the starting button is selected on DNA sequences. In the case of a barcode has already been published in journals, users can simply input it in the entry of literature information.

\section{Data collection and validation}

BioBarcode provides users with a pathway for the direct submission of their data, which can include the information related to specimens, sequences, trace files, and images. Once data are submitted, if the information needs updating, an edit function is directly accessible from the sequence and specimen pages. Projects created by any registered user (a 'project manager' status will be acquired through the project creation) will be subject to optional security measurement, and all data records will remain private to a single researcher or to a group of collaborators until they opt for public release.

BioBarcode employs a function to assess data quality (Figure 3). All submitted DNA sequences which have gone through experimental steps such as sample collection, DNA extraction, amplification, and sequencing, are translated into proteins and then compared against already known COI proteins to verify that they are true COIs using MEGABLAST program with parameters of -p $95-\mathrm{m} 8-\mathrm{e}<>-\mathrm{b}<>$. The main target database is GenBank and any in-house database can be used to be compared with the input sequence. Sequences that pass this check are then examined for stop codons (to detect the presence of pseudo-genes). If any potential errors are detected, the sequence is flagged (Figure 4). Based on these results, the blue icon shows the presence of low quality bases. Similarly, the red icon appears when the automatically translated amino acid sequence contains stop codons. The final step is uploading the sequence data into the database with specimen information.

\section{Results and discussion \\ Data access and searches}

The data access authority in BioBarcode system is based on membership level. The barcode data input and modification depend on the member level, defined as chief administrator, project manager, full member, and guest member. Any project data is accessible only when a user is registered to the project and certified by the project manager. However, up to two items, such as specimen image and species/specimen name, can be accessed by any unregistered member. Also, barcode data input is available for chief administrator, project manager, and project members. Data retrieval is restricted to project members and the chief administrator if the project is not open to the public. Full members can create and manage a project (Figure 5).

A data search can be performed by the input of a DNA sequence or a keyword. Sequence-based data retrieval is done by creating a database containing publically available mitochondrial COI gene data registered in GenBank and Korean barcode projects. The search of an input sequence data is accomplished by using the MEGABLAST program. The search results are shown in the order of sequence similarity, sequence length, and gap opening. Users can filter the results by E-value (default is 10E-5). On the same search web page, there is the keyword-based data retrieval section. Users can choose an open or a closed project type, and the barcode ID, sample ID, collector, and scientific name can be then used as a search parameter. For a historical reason of providing the service in Korea, a Korean name can be used as a search parameter. The results from both methods are directly linked to the entry of the general information of specimens.

\section{User interface}

As shown in Figure 6, the BioBarcode database consists of five entries: main menu bar, log-in system, category, 

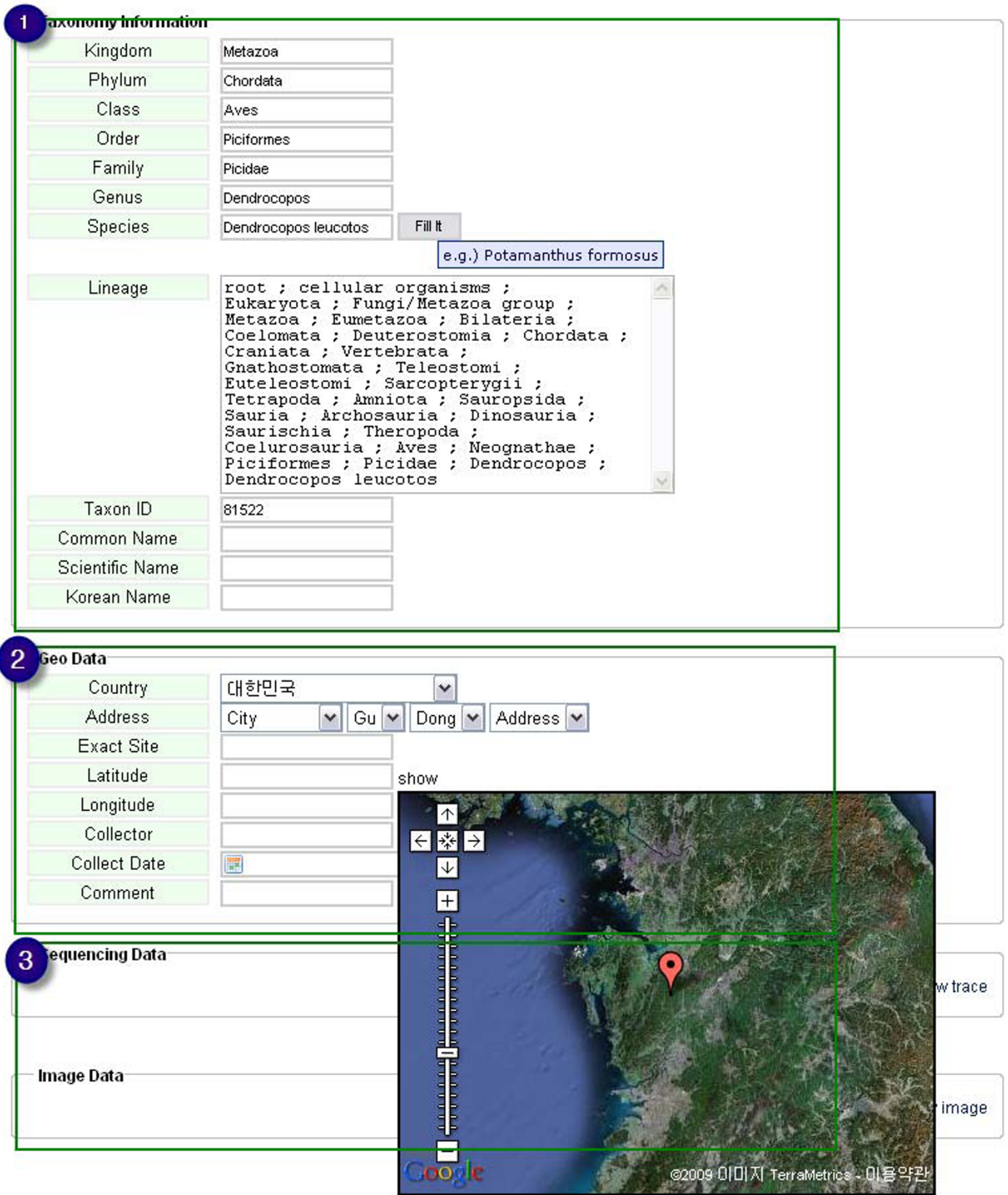

Figure 2

Screenshot of the data entry for taxonomy and geographical distribution. Clicking an icon using the Google map API makes an automatic entry of longitude and latitude of the selected location in geographical distribution. I, Taxonomy Information; 2, Geographical Information; 3, Image and Chromatogram Files. 


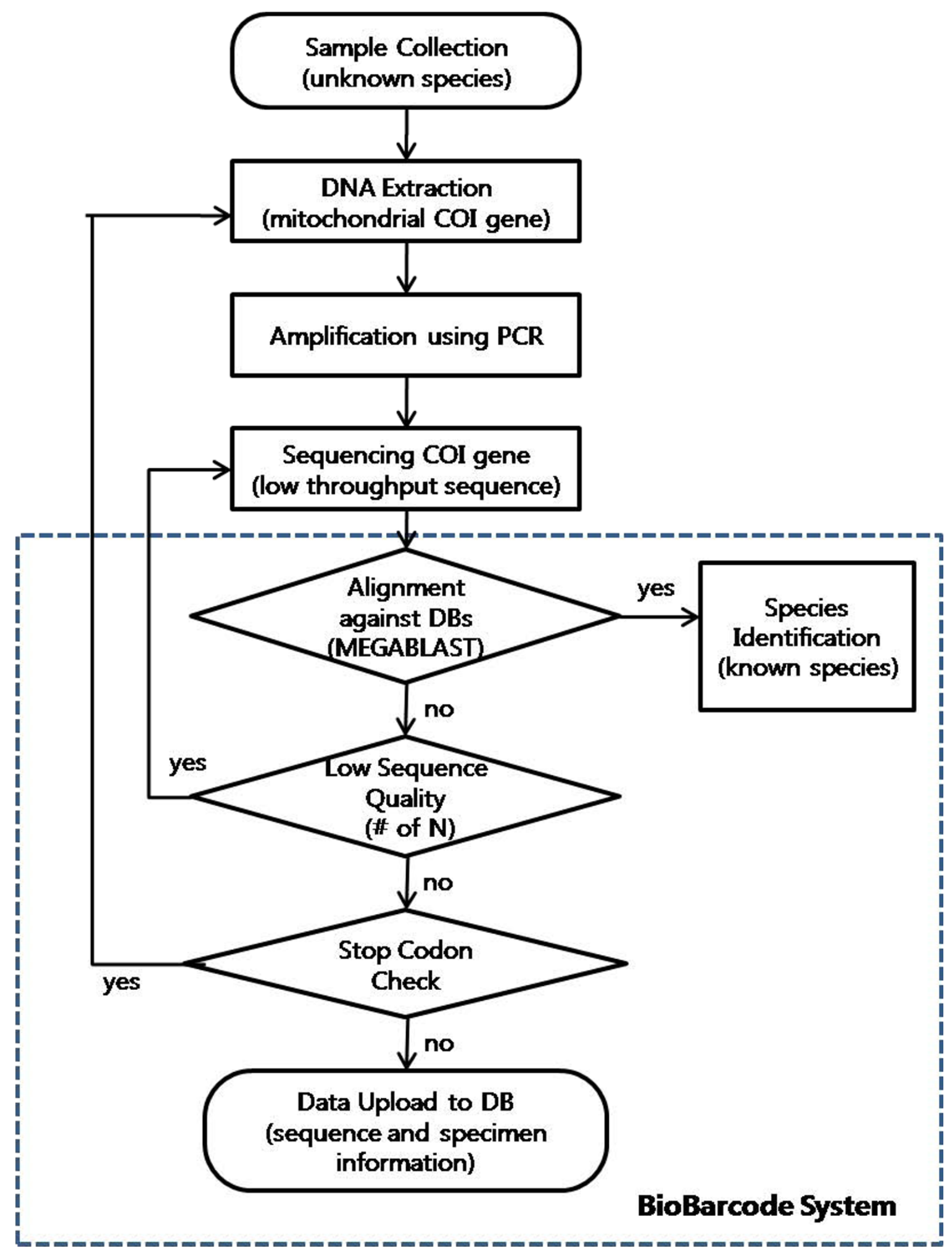

Figure 3

Flowchart of BioBarcode DNA sequence identification and quality validation. The flowchart is to explain the whole procedure from sample collection to data quality checking. The dotted line indicates how the processing occurs in BioBarcode system. 


\begin{tabular}{|c|c|c|c|c|c|c|}
\hline \multicolumn{7}{|c|}{ Entries } \\
\hline & & & \multicolumn{4}{|r|}{ Entries } \\
\hline No & Barcode & Species & Length (bp) & Data & User & Date \\
\hline 121 & KVER_04_00122 & Ardea cinerea & 667 (1) & 过@ & bird & $2009-01-21$ \\
\hline 122 & KVER_04_00123 & Ardea cinerea & \multicolumn{3}{|c|}{ Number of lowquality base: 3} & $2009-01-21$ \\
\hline 123 & KVER_04_00124 & Ardea cinerea & 664 & 电(9) & bird & 2009-01-21 \\
\hline 124 & KVER_04_00125 & Ardea cinerea & 664 & 电@ & bird & $2009-01-21$ \\
\hline 125 & KVER_04_00126 & Bubulcus ibis & 664 & 果(9) & bird & $2009-01-21$ \\
\hline 126 & KVER_04_00127 & Bubulcus ibis & $\Theta$ & Q9) & bird & 2009-01-21 \\
\hline 127 & KVER_04_00128 & Egretta alba & 664 & $\frac{p_{0} \text { Codon:3 }}{20}$ & bird & $2009-01-21$ \\
\hline
\end{tabular}

Figure 4

Screenshot of the result page showing low quality sequences and stop codons. The blue icon shows the presence of low quality bases in a sequence. Also, the red icon appears when the automatically translated amino acid sequence contains stop codons.

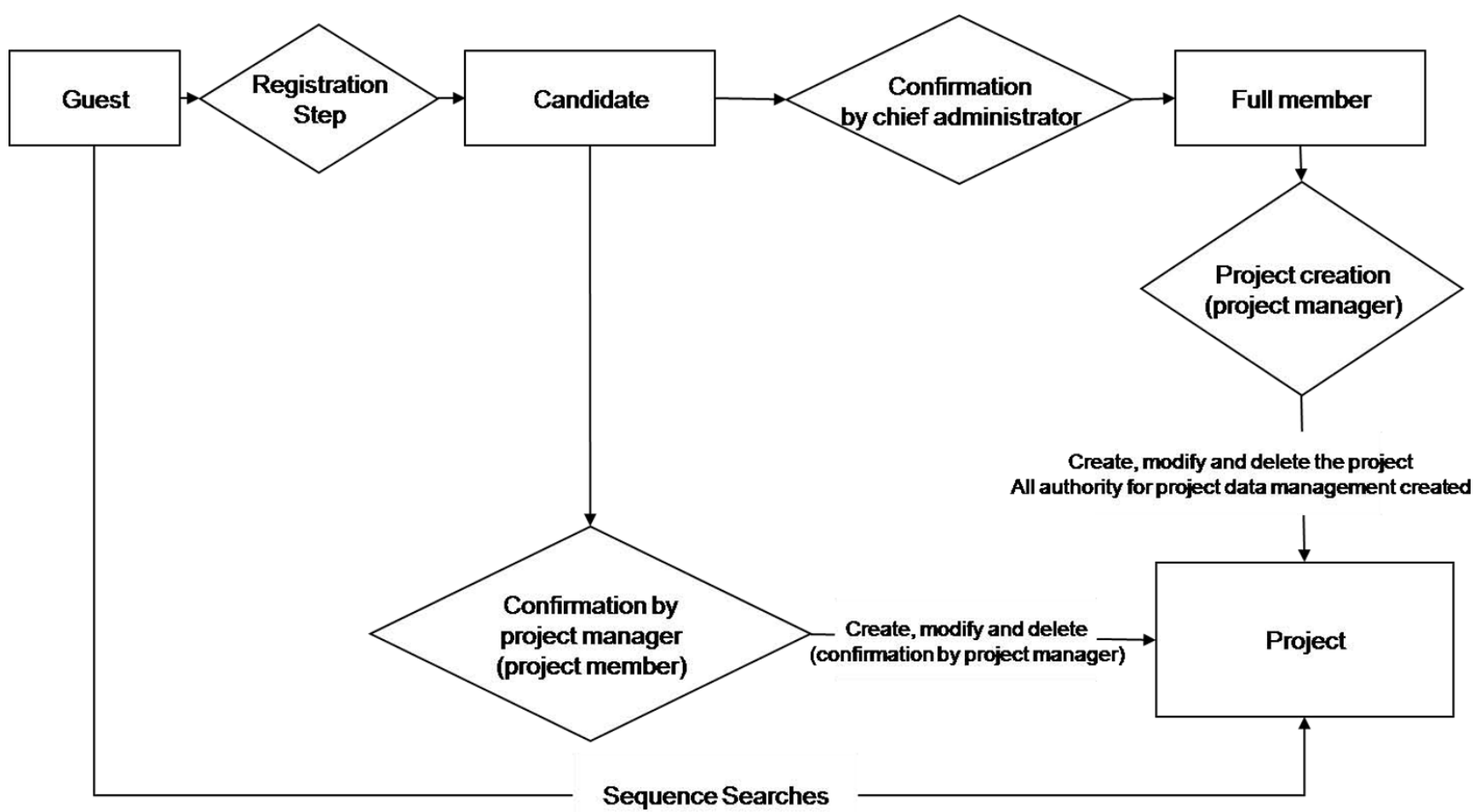

\section{Figure 5}

Data access authority and roles based on membership. The input and modification of barcode data depend on the level of members defined as chief administrator, project manager, full member, and guest member. Chief administrator, project manager, and project members only can input the barcode data. And the data retrieval is restricted to project members and the chief administrator if the project is not open to the public. 

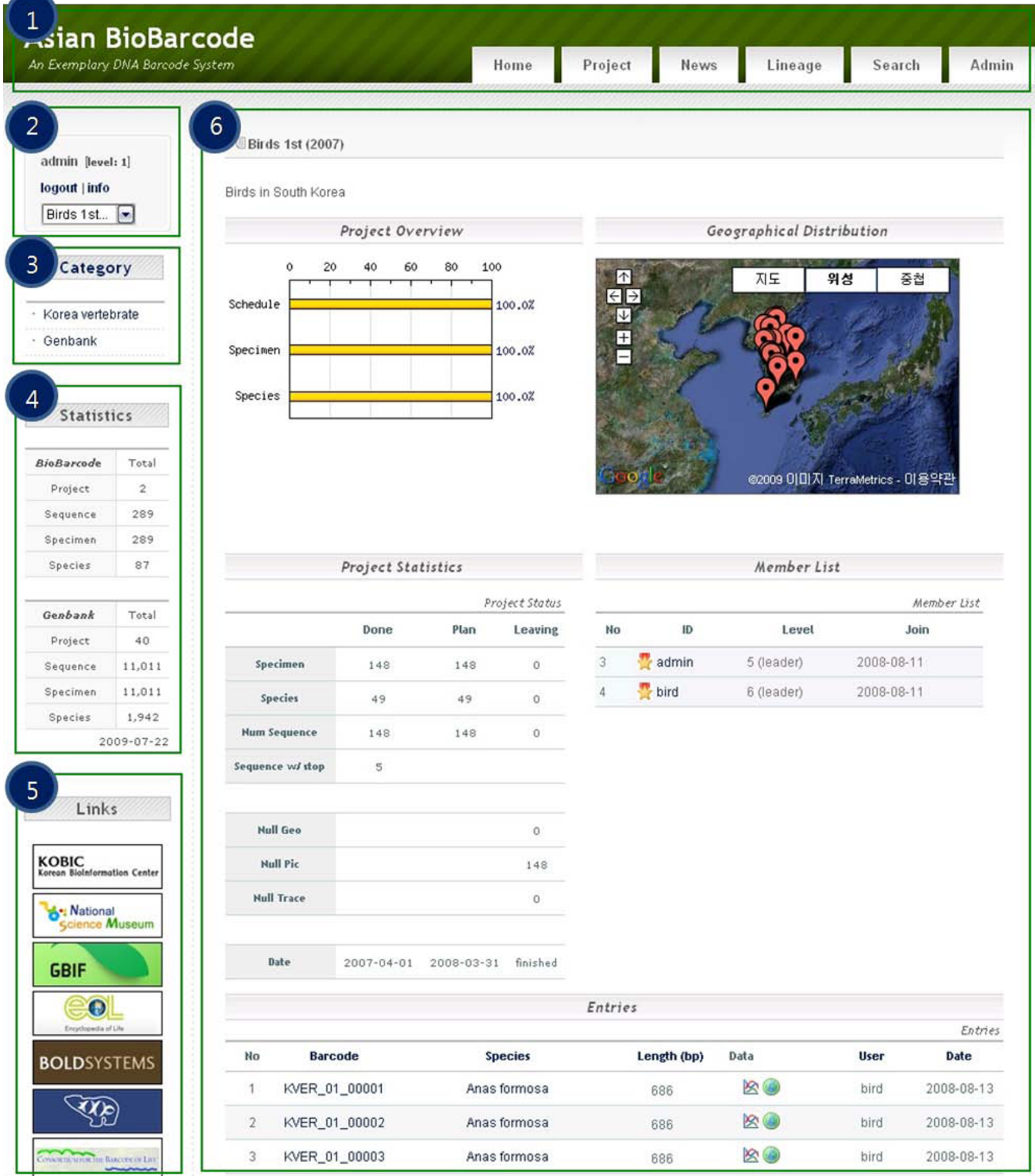

Figure 6

BioBarcode user interface. Users can search the BioBarcode DB using five entries: I, main menu bar; 2, log-in system; 3, category; 4, statistics; 5, other links. The project page (6 of Fig. 6) shows six sections such as project title, overview, geographical distribution, statistics, member list, and entries. 
statistics, and other links to related organizations to facilitate effective and user-friendly database management.

Through the 'Lineage' page of the main menu section (1 of Figure 6), taxonomy information based on input data which requires authorization can be identified. Personal information and registered project lists are available in the login state (2 of Figure 6). The 'Category' registered by administrator is linked with the 'Project' (3 of Figure 6). The statistics of registered species, specimen, sequence, projects, and trace files can be viewed on the 'Statistics' page (4 of Figure 6). The 'Project' page ( 6 of Figure 6) consists of six sections: project title, general overview (e.g., number of species, specimens and sequencing reaction, dates of start and end project,), geographical distribution, statistics, member list, and entries (e.g., barcode ID, species, and sequence with trace data).

Recently mobile interface program have become available such as Yahoo's blueprint interface http://mobile. yahoo.com/developers. We plan to implement the mobile application interface so that researchers can easily and rapidly deposit and monitor data in real time for sampling locations, collections, and observations. Another major issue is various Asian languages supports by the BioBarcode $\mathrm{DB} /$ Server construction system. We will include language pack in the next version of BioBarcode.

\section{Computational resources}

The BioBarcode system was developed with open source software. PHP and JavaScript were used for most web pages and the main system so that any future developers can join or develop their own based on BioBarcode. mySQL was used for database management and MEGABLAST was used to search sequence identity. BioBarcode does not require much computing resources, and a stand-alone PC workstation is enough to run the server. The BioBarcode server is currently optimized for IE7 internet browsers. Therefore, some javascript or ajax functions may not work correctly with other internet browsers. Internet standards will be kept more strictly in the next versions.

\section{Conclusion}

A DNA barcode server and database construction system, BioBarcode, and an exemplary server are presented, aiming to provide a platform for biological researchers who want to establish their own DNA barcode database and web server system compatible with international standards that meet the criteria in the International Nucleotide Sequence Database Collaboration (INSDC, which includes GenBank, the European Molecular Biology Laboratory, and the DNA Data Bank of Japan). BioBarcode is targeted to Asian researchers who have many local biodiversity resources. It intends to be easy to run and maintain with inexpensive open source software.

\section{Competing interests}

The authors declare that they have no competing interests.

\section{Authors' contributions}

JB directed the study and helped draft the manuscript. WK coordinated the collaboration and the study associated with BioBarcode and KBOL. CBK and WKP were involved in reviewing and critically revising it for intellectual content. SYK, SMK and HSE provided technical assistance while constructing the database. JHL conceived the study and wrote the manuscript. All authors read and approved the final manuscript.

\section{Note}

Other papers from the meeting have been published as part of BMC Bioinformatics Volume 10 Supplement 15, 2009: Eighth International Conference on Bioinformatics (InCoB2009): Bioinformatics, available online at http://www.biomedcentral.com/1471-2105/10? issue $=\mathrm{S} 15$.

\section{Acknowledgements}

The BioBarcode system was developed with funding from the Korean Research Institute of Bioscience and Biotechnology (KRIBB) Research Initiative Program, the Eco-Technopia 21 Project from Ministry of Environment of Korea (052-07I-05I), and the Ministry of Education, Science and Technology of Korea (20090080I40, 20090080I50 and MI086903000I-08N6903-00II0).

This article has been published as part of BMC Genomics Volume 10 Supplement 3, 2009: Eighth International Conference on Bioinformatics (InCoB2009): Computational Biology. The full contents of the supplement are available online at http://www.biomedcentral.com//47/-2/64//0? issue $=\mathrm{S} 3$.

\section{References}

I. Savolainen V, Cowan RS, Vogler AP, Roderick GK and Lane R: Towards writing the encyclopedia of life: an introduction to DNA barcoding. Philosophical Transactions of the Royal Society of London. Series B, Biological Sciences 2005, 360:1805-181I.

2. Hebert PDN, Cywinska A, Ball SL and deWaard JR: Biological identifications through DNA barcodes. Proc Biol Sci 2003, 270:3I3-32I.

3. Hajibabaei M, Singer GAC, Hebert PDN and Hickey DA: DNA barcoding: how it complements taxonomy, molecular phylogenetics and population genetics. TRENDS in Genetics 2007, 23(4): 167-172.

4. Miller SE: Proposed standards for BARCODE records in INSDC (BRIs). In request document for continuation of support by the Alfred P. Sloan Foundation submitted by the Smithsonian Institution on behalf of Consortium for the barcode of Life: 22 January 2006 Robert $\mathrm{H}$ 2005, 36-38.

5. Hudson ME: Sequencing breakthroughs for genomic ecology and evolutionary biology. Mol Ecol Res 2008, 8:3-17. 
6. Schuster SC: Next-generation sequencing transforms today's biology. Nat Methods 2008, 5:16-18.

7. Teletchea $F$, et al: Molecular identification of vertebrate species by oligonucleotide microarray in food and forensic samples. J Appl Ecol 2008, 45:967-975.

8. Birstein V], et al: Polyphyly of mtDNA lineages in the Russian sturgeon, Acipenser gueldenstaedtii: forensic and evolutionary implications. Conserv Genet 2000, I:8I-88.

9. Marrero P, et al: Diet of the endemic Madeira Laurel Pigeon Columba trocaz in agricultural and forest areas: implications for conservation. Bird Conserv Int 2004, 14:165-172.

10. Cristobal-Azkarate J and Arroyo-Rodriguez V: Diet and activity pattern of howler monkeys (Alouatta palliata) in Los Tuxtlas, Mexico: effects of habitat fragmentation and implications for conservation. Am J Primatol 2007, 69:1013-1029.

II. Valentini A, Pompanon F and Taberlet P: DNA barcoding for ecologists. Trends in Ecology and Evolution 2008, 24(2): I I0-II7.

12. Kristensen R, Berdal KG and Holst-Jensen A: Simultaneous detection and identification of trichothecene- and moniliformin-producing Fusarium species based on multiplex SNP analysis. J Appl Microbiol 2006, I02(4): | $07|-108|$.

13. Hebert PDN, et al: Identification of birds through DNA barcodes. PLoS Biol 2004, 2:e3 I2.

14. Ward RD, et al: DNA barcoding Australia's fish species. Philos Trans R Soc Lond B Biol Sci 2005, 360:1847-1857.

15. Meyer CP and Paulay G: DNA barcoding: error rates based on comprehensive sampling. PLoS Biol 2005, 3:e422.

16. Barrett RDH and Hebert PDN: Identifying spiders through DNA barcodes. Can I Zool 2005, 83:48I-49I.

17. Vences M, Thomas M, Meijden Van der A, Chiari $Y$ and Vieites DR Comparative performance of the I6S rRNA gene in DNA barcoding of amphibians. Frontiers in Zoology 2005, 2:5.

18. Hebert PDN, et al: Ten species in one: DNA barcoding reveals cryptic species in the neotropical skipper butterfly Astraptes fulgerator. Proc Natl Acad Sci USA 2004, I0 I: | 48 I2-| 48 I7.

19. Hajibabaei $M$, et al: DNA barcodes distinguish species of tropical Lepidoptera. Proc Natl Acad Sci USA 2006, I03:968-97I.

20. Janzen $D H$, et al: Wedding biodiversity inventory of a large and complex Lepidoptera fauna with DNA barcoding. Philos Trans R Soc Lond B Biol Sci 2005, 360:1835-1845.

21. Kress WJ, et al: Use of DNA barcodes to identify flowering plants. Proc Natl Acad Sci USA 2005, 102:8369-8374.

22. Saunders GW: Applying DNA barcoding to red macroalgae: a preliminary appraisal holds promise for future applications. Philos Trans R Soc Lond B Biol Sci 2005, 360:1879-1888.

23. Summerbell RC, et al: Microcoding: the second step in DNA barcoding. Philos Trans R Soc Lond B Biol Sci 2005, 360:1897-1903.

24. Scicluna SM, et al: DNA barcoding of blastocystis. Protist 2006, 157:77-85.

25. Sogin ML, et al: Microbial diversity in the deep sea and the underexplored 'rare biosphere'. Proc Natl Acad Sci USA 2006, 103:12115-12120.

26. Yoo HS, Eah JY, Kim JS, Kim YJ, Min MS, Paek WK, Lee H and Kim CB: DNA Barcoding Korean Birds. Mol Cells 2006, 22(3): 323-327.
Publish with Biomed Central and every scientist can read your work free of charge

"BioMed Central will be the most significant development for disseminating the results of biomedical research in our lifetime. "

Sir Paul Nurse, Cancer Research UK

Your research papers will be:

- available free of charge to the entire biomedical community

- peer reviewed and published immediately upon acceptance

- cited in PubMed and archived on PubMed Central

- yours - you keep the copyright
BioMedcentral 